\title{
Bibliografía Adicional para la Obra de la señorita Nichols
}

Esta bibliografía, que es parte de la nota precedente, no incluye estudios cuya fecha de publicación pasa de 1941, año en que apareció la bibliografía de la señorita Nichols. Por el hecho de ofrecer tan pocos títulos no conservamos el orden seguido por ella, excepto en el caso de las divisiones generales. Dentro de éstas presentamos las anotaciones en orden alfabético, sin más clasificación. Cuando hacemos referencia a un número, éste ha de entenderse como el número de una anotación en la bibliografía de la señorita Nichols.

REVISTAS ERUDITAS

1. Revista de Estudios Hispánicos (Nueva York; Río Piedras, P. R.; Madrid, 1928-1929).

Cada número de los seis publicados trae una sección bibliográfica titulada "Lengua". Esta sección tiene tres divisiones, a saber: Lenguas indias; Gramática castellana; y Lexicografía y Dialectología.

2. Revista Hispánica Moderna. (Nueva York, 1934- ).

En cada número publicado hay una sección bibliográfica, de la cual una división es Lengua con subdivisiones de Lenguas indígenas y Español. 
EL ESPAÑol americano. Estudios genERALES

3. Armas, Juan Ignacio de

Orijenes del lenguaje criollo. 2 a ed. corregida i aumentada. La Habana, 1882, $97 \mathrm{p}$.

(Cuba, Santo Domingo, Puerto Rico, Venezuela, Colombia i en alguna parte de Centro América).

4. Batres Jáuregui, Antonio

El castellano en América.

Guatemala, 1904, 286 p.

5. Beinhauer, Werner

Spaniscbe Umgangsspracbe.

Berlin - Bonn, 1930, 268 p.

6. Bertoni, Moisés Santiago

Influencia de la lengua guarani en Sudamérica y Antillas.

Asunción, 1916.

7. Bliss, G. S.

Pronóstico del tiempo con una introducción al estudio de la atmósfera y un glosario de términos meteorológicos.

Brooklyn, N. Y., 1941, 120 p.

El Glosario de términos meteorológicos (pp. 83-120), se divide en dos partes: A. Términos técnicos, pp. 83-113 y B. Términos familiares, pp. 113-120.

8. Braue, Alice

Beiträge zur Satzgestaltung der spanischen Umgangssprache. Hamburg, 1931, 123 p.

(Hamburger Studien zur Volstum und Kultur der Romanen, 7).

9. Henríquez Ureña, Pedro

"El descontento y la promesa; en busca de nuestra expresión". Boletin de la Biblioteca Nacional de Ecuador, Quito, nueva serie, Núm. 9, 125-138. 
10. Lillo, Samuel A.

Las academias hispanoamericanas y el mantenimiento de la unidad del castellano. Discurso de ingreso en la Academia Chilena.

Santiago de Chile, 1929, 51 p.

11. Lugones, Leopoldo

"La lengua que hablamos".

La Nación, Buenos Aires. 28 agosto 1927. (Véase 184).

12. Méndez Bejarano, $M$.

Conversación familiar acerca de la prosodia española en Castilla, Andalucia y Ultramar.

Alcalá de Henares, 1927, 27 p.

13. Menéndez Pidal, Ramón

Manual de gramática bistórica española. Sexta edición corregida y aumentada.

Madrid, 1941, 369 p.

Otras ediciones: Primera, Madrid, 1904, 233 p.; Segunda, M., 1905; Tercera, M., 1914, 269 p.; Cuarta, M., 1918, 299

p.; Quinta, M., 1925, 325 p.

14. Nelson, Ernesto

The Spanish American Reader.

New York, 1916, 367 p.

ESTUDIOS GENERALES DE ASPECTOS ESPECÍFICOS

Lexicografía y semántica

15. Mahn, Karl August Friedrich

Etymologische Untersucbungen auf dem Gebiete der Romanischen Spracben.

Berlin, 1855, $192 \mathrm{p}$.

16. Méndez Percira, Octavio

Significado peyorativo de los nombres formados con terminaciones que presentan la letra u. Ensayo filológico.

Santiago de Chile, 1912, $70 \mathrm{p}$. 
17. Monner Sans, Ricardo

Asnología-Vocabulario y refranero.

Buenos Aires, 1921, 96 p.

18.

Can y perro.

Buenos Aires, 1919, 8 p.

19

El neologismo.

Buenos Aires, 1906, 22 p.

20

La religión en el idioma; ensayo paremiológico.

Buenos Aires, 1899, $253 \mathrm{p}$.

21.

Perrología. El perro a través âlel diccionario y del refranero.

Buenos Aires, 1923, 168 p.

\section{Ortografia}

22. Amunátegui Reyes, M. L.

Ortografia razonada.

Santiago de Chile, 1926, 63 p.

23.

Uso de la $G$ i de la J. Representación becha ante la Real Academia Española.

Santiago de Chile, 1920, 13 p.

24. Cabezón, Carlos

Libros, folletos y articulos impresos con ortografia rrazional.

Killota (Chile), 1909, 31 p.

25

Notas sobre la reforma ortográfiqa por...

Santiago de Chile, 1892, 67 p. 
26. García de San Vicente, Nicolás

Ortografía española acomodada a la pronunciación megicana, puesta en verso e ilustrada con muchas reglas nuevas.

Mégico, 1853, $104 \mathrm{p}$.

Kabezón, Kárlos véase Cabezón, Carlos.

27. Llaverias, Federico

Breves apuntes sobre ortografía castellana.

Camagüey, 1907, $61 \mathrm{p}$.

Qabezón, Qárlos véase Cabezón, Carlos.

\section{Fonética}

28. Lenz, Rodolfo

"Necesidad de celebrar una conferencia fonética internacional para la adopción de un alfabeto fonético universal. Discurso leído en la sesión del IV Congreso de Ciencias Naturales y Antropológicas". Revista Cbilena de Historia Natural, XIII, 1909, 25-32.

\section{AMERICANISMOS}

Diccionarios y vocabularios

29. Costa Alvarez, Arturo

El diccionario ideológico de la lengua.

Buenos Aires, 1927.

30. Cuervo, Rufino José

Diccionario de construcción y régimen de la lengua castellana.

París, 1886-93, 2 tomos: I A-B; II C-D.

31. Lenz, Rodolfo

Problemas del diccionario castellano en América.

Buenos Aires, 1927, 47 p.

32. Retortillo y Tornos, Alfonso

Diccionario histórico-popular bispanoamericano.

Madrid, s. a., 580 p. 
33. Rodríguez-Navas, $M$.

Diccionario manual de la lengua española. Contiene todas las voces corrientes $y$ familiares en España, en las Republicas Hispanoamericanas y en Filipinas, $y$, además, las palabras técnicas usuales de Ciencias, Artes e Industrias, o sea todos los artículos comprendidos en el Diccionario de la Real Academia y veinticinco mil voces más.

Madrid, s. a., 1693 p.

34. Vergara Martín, Gabriel

Diccionario de frases, adagios, proverbios, modismos, locuciones y frases proverbiales que se emplean en la América espanola o que se refieren a ella.

Madrid, 1929, 149 p.

35.

Diccionario bispanoamericano de voces sinónimas y análogas. Madrid, 1930,285 p.

36. Vizuete, Pelayo (redactor en jefe)

Diccionario tecnológico bispanoamericano. Redactado por la Unión Internacional Hispanoamericana de Bibliografía y Tecnologia Cientificas.

Madrid, 1926.

\section{Toponimia}

37. Pector, Désiré

Considérations sur quelques noms indigènes de localités de l'stbme Centre-Américain.

Paris, 1892, 23 p.

38. Vergara Martín, Gabriel

Diccionario etnográfico americano, contiene las denominaciones de gran numero de naciones, tribus y pueblos de indios; los patronímicos de los naturales de los estados americanos $y$ de los de algunas comarcas, regiones, provincias y localidades de ellos, $y$ los nombres particulares con que se designan en distintos estados los indigenas y los extranjeros, segun su clase y condición.

Madrid, 1922, 246 p. 
SECCIONES NACIONALES

Argentina

39. Borges, J. L.

"El idioma de los argentinos".

La Prensa, Buenos Aires, 24 septiembre 1927.

(Véase 403).

40. Capdevila, Arturo

"El tú y el vos en América".

La Prensa, Buenos Aires, 20 febrero 1927.

(Véase 405).

41 .

"La prodigiosa y díscola ciudad del idioma común".

La Prensa, Buenos Aires, 12 junio 1927.

(Véase 405).

42. Castro, Américo

"En torno al posible idioma argentino".

La Nación, Buenos Aires, 18 septiembre 1927.

43.

"¿Por qué desean ciertos argentinos una lengua nacional?"

El Sol, Madrid, 22 y 25 de septiembre 1927.

44. Costa Alvarez, Arturo

"La obra de Monner Sans en nuestra lengua".

Nosotros, 1927, LVI, 350-356.

45. Ghiraldo, A.

"La utopía de un idioma propio".

El Sol, Madrid, 25 agosto 1927.

("Españolidad" y "argentinidad". Una encuesta de "crítica").

46. Lehmann-Nitsche, Roberto

"El idioma chechehet (Pampa Bonaerense). Nombres propios".

Revista del Museo de La Plata, xxxm, 277-291. 
47. Lehmann - Nitsch, Roberto

"Etimologías españolas: gaucho".

La Prensa, Buenos Aires, 6 febrero 1927.

48. Leguizamón, Martiniano

El origen de las boleadoras y el lazo.

Buenos Aires, 1919, 53 p.

(Publicaciones de la Facultad de Filosofía y Letras. Sección Antropología, Núm. 19. Etnografia del Plata).

49. Lorenzo, $\mathrm{T}$.

"Los nuevos horizontes del idioma".

La Nación, Buenos Aires, 13 noviembre 1927.

(Véase 419).

50. Maldones, Estanislao

Los bombres venidos del norte. Traducción quechua. Estudio

a propósito de los nombres catamarqueños "Sumalao", "Pilciao", "Fiambola", "Amanao", "Colalao" y el americano "Los Andes".

Catamarca, 1911,36 p.

51. Pascual Ramírez, Pedro

Los Huarpes. Etimologias de las palabras usadas por el pueblo. Buenos Aires, 1938, 244 p.

52. Ragucci, Rodolfo

Palabras enfermas y bárbaras.

Rosario, 1941, 247 p.

\section{COLOMBIA}

53. Lanao, Manuel E.

Apuntaciones criticas sobre el idioma castellano. Provincialismos de Riobacha.

Santa Marta, 1920.

54. Marroquín y Osorio, José Manuel

Discursos académicos y otros escritos sobre filología y corrección del lenguaje.

Bogotá, 1929, 255 p. 
55. Pérez Arbeláez, Enrique

Plantas medicinales de Colombia.

Bogotá, 1937, 295 p.

COSTA RICA

56. Bolívar Coronado, Rafael

Parnaso costarricense. Selección esmerada de los mejores poetas

de Costa Rica, por...

Barcelona, 1921, 199 p.

Vocablos costarricenses, pp. 187-199.

57. Noriega, Félix

Diccionario geográfico de Costa Rica.

San José, 1904, 247 p. (Segunda edición: San José, 1923, 274 p.)

58. Pittier de Fábrega, Henri François

Nombres geográficos de Costa Rica I Talamanca (primera contribución).

San José, 1895, 46 p.

59. Quesada S., Napoleón

Lecciones de gramática castellana.

San José, 1935, 412 p. (Tercera edición).

(Contiene frecuentes alusiones al uso costarricense).

60. Sotela, Rogelio

Palabras y locuciones mal empleadas en Costa Rica. (Complemento gramatical de los programas de castellano).

San José, 1928, 69 p. (Tercera edición: San José, 1941).

61. Standley, Paul C.

Flora of Costa Rica.

Chicago, 1937, 2 tomos. 
62. Marinello Vidaurreta, $\mathrm{J}$.

"Un guacalito de cubanismos".

Archivos del Folklore Cubano, 1927, II, 363-368; 1928, III, 21-26.

63. Perdomo, José P.

Léxico tabacalero cubano.

La Habana, 1940.

64. Portell Vilá, Herminio

"Nomenclatura monetaria cubana".

Arcbivos del Folklore Cubano, 1927, II, 393-394.

65. Roig y Mesa, Juan Tomás

Diccionario botánico de nombres vulgares cubanos.

La Habana, 1928, 3 tomos.

66. Toor, Frances

Spanish for your Mexican and Cuban visits.

Mexico, 1941, 251 p.

\section{CII工 E}

67. Bañados, Guillermo $M$.

Apuntes para un diccionario marítimo militar chileno.

Santiago, 1923, 287 p.

68. Latorre, Mariano

La literatura de Chile.

Buenos Aires, 1941, 206 p.

69. Laval Alvear, Ramón A.

"Del latín en el folklore chileno".

Anales de la Universidad de Chile, 1909, pp. 931-952.

"Un arsenal de frases, refranes, versos, anécdotas, etc., en que se encuentran vestigios de un latín casi siempre inventado y divertido". Rojas Carrasco (Filología chilena, 264). 
70. Laval Alvear, Ramón A.

Paremiologia chilena.

Santiago, 1923, 96 p. (Segunda edición: Santiago, 1928, 95 p.)

71. Lenz, Rodolfo

"Influencia de la cultura indígena en el pueblo chileno".

Trabajo presentado al VIII Congreso general chileno celebrado en Temuco del 12 al 23 de febrero de 1913.

72.

La oración y sus partes. Estudios de gramática general y castellana.

Madrid, 1920, 545 p. (Segunda edición: Madrid, 1925, 558

p.; Tercera edición: Madrid, 1935, 570 p.)

(Contiene muchas referencias al español de Chile).

73. Paulsen, Fernando

Reparos de reparos, o sea lijero examen de los Reparos al Diccionario de Cbilenismos de don Zorobabel Rodriguez, por don Fidelis P. del Solar.

Santiago, 1876,35 p.

(Véanse 697 y 706).

74. Reed, Edwin C.

"Catálogo de las aves chilenas".

Anales de la Universidad de Cbile, xcm, pp. 197-213.

75.

"Catálogo de los peces chilenos".

Anales de la Universidad de Cbile, xcvir, pp. 653-673.

76. Ruiz, Hipólito

Florae peruvianae, et chilensis prodomus; sive, novarum generum plantarum peruvianarum, et cbilensium descriptiones, et icones, ... Ed. 2.

Roma, 1797, 151 p. 
77. Cordero, Luis

Enumeración botánica de las principales plantas, así útiles como nocivas, indigenas o aclimatadas, que se dan en las provincias del Azuay y de Cañar de la República del Ecuador. Cuenca, 1911, 304 p.

78. Cordero Palacios, Alfonso

Léxico de vulgarismos azuayos.

Ecuador, 1928.

ESTADOS UNIDOS

79. Bailey, Florence M.

Birds of New Mexico.

Washington, D. C., 1928, 807 p.

80. Bailey, Vernon

"Life Zones and Crop Zones of New Mexico".

North American Fauna, No. 53. United States Biological Survey.

Washington, D. C., 1913, 100 p.

81

"Mammals of New Mexico".

North American Fauna, No. 53. United States Biological' Survey.

Washington, D. C., 1913, 412 p.

82. Barnes, W. C.

Arizona Place Names.

Tucson, 1935, $503 \mathrm{p}$.

83. Espinosa, Aurelio Macedonio

"Speech Mixture in New Mexico".

The Pacific Ocean in History.

New York, 1917, pp. 408-428. 
84. Fortier, Alcée

"The isleños of Louisiana" en

Louisiana Studies.

New Orleans, 1894, pp. 194-210.

85. Kiddle, Lawrence B.

"Los nombres del pavo en el dialecto nuevomejicano".

Hispania, 1941, xxIv, 2; pp. 213-216.

86. Wooton, Elmer O. and Standley, Paul Carpenter

Flora of New Mexico.

Washington, D. C., 1915, 794 p.

GUATEMAIA

87. Fuentes y Guzmán, Francisco Antonio de

"Flora de Guatemala".

Anales de la Sociedad de Geografía e Historia, Ix, 3, pp. 370374.

88. Morescier, Alfredo

El último maya. Principe Kicbé.

Guatemala, 1936, 210 p.

(Vocabulario, pp. 204-210).

89. Roque, José María

"Flora médica guatemalteca".

Anales de la Sociedad de Geografia e Historia, I, 2, pp. 198207.

H ONDURAS

90. Schuller, Rodolfo R.

Maya-K'icé Studien. I Der Name lenca. Nebst einigen Bemerkungen über verschiedene geographische Ortsnamen von Honduras, Mittelamerika.

Separat-abdruck aus: Internationales Arcbiv für Etnografie, Bd. 30, Heft 4-5, pp. 95-118. 
91. Standley, Paul Carpenter.

Flora of the Lancetilla Valley, Honduras.

Chicago, 1931,418 p.

92. Tobias Rosa, J. M.

Flora y fauna santabarbarense.

Tegucigalpa, 1918, $73 \mathrm{p}$.

MEXICO

93. Amador, Elías

Nombres indigenas todavía en uso en el Estado de Zacatecas.

Zacatecas, $1897,66 \mathrm{p}$.

94 Anónimo

"Noticia sobre la lengua maya; origen de los nombres de algunas ciudades de Yucatán”.

El Nuevo Yucatán, Mérida, $1^{\circ}$ febrero 1926, p. 42.

95.

Suma de visitas de pueblos por orden alfabético.

Ms. 2,800 de la Biblioteca Nacional de Madrid. Anónimo de la mitad del siglo XVI.

Madrid, $1905,332 \mathrm{p}$.

96. Asiain, Lamberto

División municipal y prontuario geográfico de la Repriblica Mexicana.

México, 1908, 390 p.

(La parte iI da los nombres de lugar con su sentido en los dialectos locales).

97. Atl, Dr. (seudónimo de Gerardo Murillo)

Las artes populares, segunda edición.

México, 1922, 2 vols. (293 p. y 292 p.)

(Terminología charrera, pp. 243-260 del vol. II).

98. Azuela, Mariano

Los de abajo.

New York, 1939, 181 p. 
(Texto escolar preparado por J. E. Englekirk y L. B. Kiddle y destinado al uso de los estudiantes de español en los colegios y universidades angloparlantes).

Notes on the Language of Los de abajo, pp. xlix-li.

Vocabulary, pp. 125-181.

99. Blake, S. F.

"A flora of Mexico".

Science, vol. 74, Núm. 1923 (Nov. 6, 1931).

100. Bolio Ontiveros, Edmundo

Mayismos, barbarismos y provincialismos yucatecos.

Mérida, 1931, 55 p.

101. Carreño, Alberto María.

La lengua castellana en México.

México, 1925.

102. Castañeda, Alfonso Manuel

"La flora del Estado de Jalisco".

Boletín de la Junta Auxiliar jalisciense de la Sociedad Mexicana de Geografía y Estadística. Núm. 5, octubre 1933.

103. Códice Martínez Gracida

Códice mixteco. Lienzo de Zacatepec publicado por el Dr. Antonio Peñafiel.

México, 1900.

Nombres geográficos de México-Jamiltepec.

104. García Conde, Angel

"Etimología geográfica nacional".

Investigaciones lingüisticas, II, núms. 3-4, pp. 335-339 (juliooctubre 1934).

105. Ibarra de Anda, F.

Geonimia indigena mexicana.

México, 1932, 114 p.

106. Inurreta, José Luis

Cosas de mi pueblo. Bocetos de costumbres populares del Estado de Tabasco. 
México, 1924, $111 \mathrm{p}$.

(Vocabulario de los regionalismos que figuran en este libro, pp. 105-111).

107. Levy, Bernard

Present Day Spanish.

New York, 1940, 360 p. (Segunda edición: New York, 1941, $376 \mathrm{p.}$ )

(Glossary of Mexicanisms, pp. 282-287).

108. Martínez Cantón, Eduardo

Etimología de las palabras con que son conocidos algunos puntos geográficos mayas apuntados de acuerdo con los vocabularios mayas.

Extracto de "Yucatán fotográfico", junio, 1929, $15 \mathrm{p}$.

109. O'Gorman, Edmundo

Breve bistoria de las divisiones territoriales; aportación a la bistoria de la geografía de México.

México, 1937, 261 p.

Indice de nombres geográficos, pp. 221-234.

110. Olaguíbel, Manuel de

La ciudad de México y el Distrito Federal. Toponimia azteca.

Toluca, 1898, $121 \mathrm{p}$.

111.

Onomatología del Estado de México, comprendiendo cuatro idiomas: mexicano, otomi, mazabua y tarasco. Con un apéndice relativo a bistoria, arqueología y a la flora $y$ a la fauna del Estado.

Toluca, $1894,210 \mathrm{p}$.

112. Peñafiel, Antonio

Nomenclatura de México.

México, 1897. En dos partes: $1^{\mathrm{a}}$ parte, 224 p.; $2^{\mathrm{a}}$ parte, diccionario, $336 \mathrm{p}$.

113. Pierce, Frank Cushman

Colloquial and Idiomatic Mexican.

Brownsville, Texas, 1916, $64 \mathrm{p}$. 
114. Rosa, Agustín de la

Aztec Place-Names; their meaning and mode of composition. Translated from the Spanish of Padre Agustin de la Rosa and Dr. Antonio Peñafiel by Frederick Starr.

Chicago, 1895, $12 \mathrm{p}$.

115. Roviroso, José Narciso

Nombres geográficos del Estado de Tabasco o datos para un diccionario etimológico tabasqueño-chiapaneco.

México, $1888,36 \mathrm{p}$.

116. Santamaría, Francisco J. y Domínguez, Rafael

Ensayos criticos de lenguaje.

México, 1940, 302 p.

117. Secretaría de Agricultura y Fomento de México.

Catálogo alfabético de nombres vulgares y científicos de plantas que existen en México.

México, 1923.

118. Secretaría de la Economía Nacional. Departamento del Petróleo. Vocabulario inglés-español de la industria petrolera.

México, 1939, 65 p.

119. Seler, Eduard

Altmexikanische Studien.

Berlin, 1890, 188 p.

120.

Gesammelte Abbandlungen zur Amerikaniscben Spracb- und Altertbumskunde.

Berlin, 1902-1923, 5 vols.

(Hay un Wort- und Sachregister zum ersten, zweiten und dritten Bände de esta publicación. Berlín, 1914, 370 p.)

121. Sessé, Martín

Flora mexicana a Martino Sesse et Josepho Marianno Mociño exarata.

México, 1894. Ed. 2, 240 p. (1* edición: México, 1887). 
122. Standley, Paul Carpenter

Flora of Yucatán.

Chicago, 1930,492 p.

123. Staub, Walther

"Zur Kenntnis der Indianischen Ortsnamen in der Huaxteca (Ost-Mexiko)".

Zeitschrift der Gesellschaft für Erdkunde zu Berlin, 1924, pp. $215-234$.

$$
\text { NICA R A G U A }
$$

124. Barreto, Mariano

Idioma y letras.

León de Nicaragua, 1902-1904, 2 vols.

$$
\text { PERU }
$$

125. Palomino Araña, Heli

Tablas ortográficas, metodologia y puntuación.

Lima, Perú, 1938.

126. Romero, Fernando

12 novelas de la selva.

Lima, 1934, 147 p.

(Vocabularios: pp. 55-56; 61-62;68; 73-74; 81-82; 88;

$101 ; 113 ; 120-121 ; 133-135 ; 146-147)$.

127. Ruiz, Hipólito

Véase el número 76 de esta bibliografia.

PUERTO RICO

128. Stahl, Agustín

Estudios sobre la flora de Puerto Rico. Segunda edición.

San Juan, 1936-1937, 3 vols.

\section{REPUBLICA DOMINICANA}

129. Jiménez, Ramón Emilio.

Del lenguaje dominicano. 
Ciudad Trujillo, 1941.

(Publicación núm. 3. Academia Dominicana de la Lengua).

130. Patín Maceo, Manuel A.

"Americanismos en el lenguaje dominicano".

Anales de la Universidad de Santo Domingo, rv, fascs. III-rv, pp. 409-423, julio-diciembre 1940.

SALVADOR, EL

131. Barberena, Santiago Ignacio

"Estudios de toponimia salvadoreña".

Repertorio salvadoreño, vIr, núms. 3-4, pp. 110-122; $159-$

165; noviembre-diciembre 1892.

132. Garcia, Miguel Angel

Diccionario bistórico-enciclopédico de la República de El Salvador.

San Salvador, 1928-1929, 3 vols.

133. Ministerio de Instrucción Pública.

Flora salvadoreña.

San Salvador, 1926, 5 vols.

(Contiene 500 ilustraciones de plantas salvadoreñas con sus nombres vulgares y científicos).

134. Salarrué (seudónimo de Salvador Salazar Arrué)

Cuentos de barro.

San Salvador, $1934,180 \mathrm{p}$.

(Vocabulario de modismos del lenguaje cuscalleco empleados en este libro, pp. 171-180).

135. Standley, Paul Carpenter y Salvador Calderón

Lista preliminar de las plantas de El Salvador.

San Salvador, 1925, 274 p.

VENEZUELA

136. Jahn, Alfredo

Las palmas de la flora venezolana.

Caracas, 1908, 126 p. 
137. Jahn, Alfredo

"Nombres geográficos indígenas de Venezuela".

Cultura venezolana, xIv, 113, pp. 138-142; julio 1931.

138. Landaeta, Federico

Rastrillo. (Relatos de un antro de horrores).

Caracas, 1939, 139 p.

"Algunas frases criollas y de argot del hampa que figuran en

el presente libro", pp. 131-135.

139. Machado, José E.

Cancionero popular venezolano. Cantares y corridos. Galerones y glosas. Con varias notas geográficas, bistóricas y lingiiisticas para explicar o aclarar el texto.

Caracas, 1919, 251 p.

140. Picón-Salas, Mariano

Odisea de Tierra Firme.

Santiago de Chile, 1940, 139 p. Segunda edición.

Glosario, pp. 135-139.

141. Uslar Pietri, Arturo

Las lanzas coloradas.

Santiago de Chile, 1940, 181 p.

Vocabulario de venezolanismos, p. 181.

Lawrence B. Kiddele,

Universidad de Tulane. 